\title{
THE COMPLETE IRREDUCIBLE SYSTEM OF INVARIANTS OF THREE QUADRICS
}

\author{
by T. SCOTT
}

(Received 1st June 1961)

\section{General}

The present account is an application of the principles of combinantal forms and Schur function analysis given in a previous paper $(A)$, the references therein being henceforward denoted by $\mathrm{A} 1$ to $\mathrm{A9}$, and the complete irreducible system of invariants of three quaternary quadrics will now be obtained from the complete system (not necessarily itself irreducible) derived by Turnbull (A5, p. 483). This latter system comprises 47 invariants, viz. 15, 1, 6, 6, 1, 15 and 3 members of total degrees $4,6,8,10,12,14$ and 18 respectively in the coefficients of the quadrics. It will be proved that all of these are irreducible except for the one of degree 12 and the three of degree 18, the former being of especial interest as it is a real combinant and moreover, involves unusual features in the proof of its reduction and also in the derivation of the form expressing it in terms of irreducible invariants.

\section{Associated Combinantal Forms}

The following table lists the invariants tabulated in A5 rearranged in such a way as to show the relevant combinantal forms, in terms of whose coefficients the invariants can be linearly expressed, but on the initial assumption that all members of the system are irreducible. In the case of $N=14$, for example, if the $(6,6,2)$ form were found to be reducible then generalised combinants such as $P(\alpha \beta \gamma)^{3}(q r \xi)^{2} p_{v}, P(\alpha \beta \gamma)^{4} p_{\lambda}^{2}, P(\alpha \beta \gamma)^{4}(q r \xi)$ would have to be considered in turn, since all coefficients of $P(\alpha \beta \gamma)^{2}(q r \xi)^{4}$ would be reducible.

Obviously all invariants of total degrees 4 and 6 are irreducible, and so it remains only to apply $S$-function analysis (A4) to types which appear as end coefficients of combinantal forms in respect of the remaining members in Table (i).

\section{Invariants of Degree 8}

The total number $v$ of linearly independent invariants (reducible and irreducible) of partial degrees $(4,2,2)$ is the coefficient of $\left\{4^{4}\right\}$ in the expansion:

$$
[\{2\} \otimes\{4\}][\{2\} \otimes\{2\}]^{2}=\left[\{8\}+\{62\}+\left\{4^{2}\right\}+\left\{42^{2}\right\}+\left\{2^{2}\right\}\right]\left[\{4\}+\left\{2^{2}\right\}\right]\left[\{4\}+\left\{2^{2}\right\}\right] \text {. }
$$

It is found that $v=6$ given by the Young Tableaux corresponding to the products:

$$
\left\{4^{2}\right\}\{4\}^{2} ;\left\{4^{2}\right\}\left\{2^{2}\right\}^{2} ;\left\{42^{2}\right\}\{4\}\left\{2^{2}\right\} ;\left\{42^{2}\right\}\left\{2^{2}\right\}\{4\} ;\left\{42^{2}\right\}\left\{2^{2}\right\}^{2} ;\left\{2^{4}\right\}\left\{2^{2}\right\}^{2},
$$


whereas the number $v^{1}$ of reducible types is 5 , viz.

$(4,0,0)(0,2,2) ;(3,1,0)(1,1,2) ;(3,0,1)(1,2,1) ;(2,2,0)(2,0,2) ;(2,1,1)(2,1,1)$ and so there is one $(4,2,2)$ invariant, as given in the complete system. Also from Table (i) it is seen that $P(\alpha \beta \gamma)^{2} p_{\lambda}^{2}$ must exist with its leading term irreducible

TABLE (i)

\begin{tabular}{|c|c|c|c|c|}
\hline $\begin{array}{c}\text { Total } \\
\text { degree } \\
N\end{array}$ & $\begin{array}{c}\text { Invariant } \\
\text { type }\end{array}$ & $\begin{array}{l}\text { No. of } \\
\text { invariants }\end{array}$ & $\begin{array}{l}\text { Type of } \\
\text { combinantal } \\
\text { form }\end{array}$ & $\begin{array}{c}\text { Coeffs.: } \\
\text { partial degrees } \\
(m, n, p)\end{array}$ \\
\hline 4 & $\begin{array}{l}a_{\alpha}^{2} \\
b_{\alpha}^{2} \\
(A B)^{2} \\
(A b c)^{2}\end{array}$ & $\begin{array}{l}3 \\
6 \\
3 \\
3\end{array}$ & $P p_{\lambda}^{4}$ & $\begin{array}{l}(4,0,0) \\
(3,1,0) \\
(2,2,0) \\
(2,1,1)\end{array}$ \\
\hline 6 & $\Phi_{123}=(A B)(B C)(C A)$ & 1 & $P(\alpha \beta \gamma)^{2}$ & $(2,2,2)$ \\
\hline 8 & $\begin{array}{l}(B C a \alpha)^{2} \\
(A \beta \gamma)^{2}\end{array}$ & $\begin{array}{l}3 \\
3\end{array}$ & $P(\alpha \beta \gamma)^{2} p^{2}$ & $\begin{array}{l}(4,2,2) \\
(3,3,2)\end{array}$ \\
\hline 10 & $\begin{array}{l}(A b c)(A \beta \gamma) b_{\gamma} c_{\beta} \\
(A b c)(B c a)(A B) a_{\gamma} b_{v}\end{array}$ & $\begin{array}{l}3 \\
3\end{array}$ & $P(\alpha \beta \gamma)^{2}(q r \xi)^{2}$ & $\begin{array}{l}(2,4,4) \\
(3,3,4)\end{array}$ \\
\hline 12 & $\Omega=\left(\begin{array}{ccc}a & c & b \\
\beta & \alpha & a\end{array}\right)$ & 1 & $P(\alpha \beta \gamma)^{4}$ & $(4,4,4)$ \\
\hline 14 & $\begin{array}{l}(A b c)(B c a)(A B)\left({ }^{a} c_{\beta}{ }^{b}\right) \\
(A b c)(B \gamma \alpha)(A C)(C B) b_{\gamma} c_{\alpha} \\
(A b c)(A \beta \gamma)\left({ }_{\alpha}^{b}\right)\left({ }_{\beta}^{a}{ }_{\gamma}\right) \\
(A \beta \gamma)(B \gamma \alpha)(A B) c_{\alpha} c_{\beta}\end{array}$ & $\begin{array}{l}3 \\
6 \\
3 \\
3\end{array}$ & $P(\alpha \beta \gamma)^{2}(q r \xi)^{4}$ & $\begin{array}{l}(6,6,2) \\
(6,5,3) \\
(6,4,4) \\
(5,5,4)\end{array}$ \\
\hline 18 & $(A \beta \gamma)(B \gamma \alpha)(A B)\left({ }^{a} b^{\gamma} a^{\beta}\right)$ & 3 & $P(\alpha \beta \gamma)^{6}$ & $(6,6,6)$ \\
\hline
\end{tabular}

and thus the $(3,3,2)$ type is also irreducible. The six invariants of total degree 8 are therefore all irreducible.

\section{Invariants of Degree 10}

In this case the total number of invariants of type $(2,4,4)$ is the coefficient of $\left\{5^{4}\right\}$ in the expansion:

$$
[\{2\} \otimes\{4\}]^{2}[\{2\} \otimes\{2\}]=\left[\{8\}+\{62\}+\left\{4^{2}\right\}+\{42\}^{2}+\left\{2^{4}\right\}\right]^{2}\left[\{4\}+\left\{2^{2}\right\}\right]
$$

and $v=2$ arising from the products $\left\{4^{2}\right\}^{2}\left\{2^{2}\right\}$ and $\left\{42^{2}\right\}^{2}\left\{2^{2}\right\}$. Since there is only one reducible combination- $-(2,2,2)(2,2,0), v^{1}=1$, which proves that the one $(2,4,4)$ invariant in Table (i) is irreducible. 
Again, from the combinantal form $P(\alpha \beta \gamma)^{2}(q r \xi)^{2}$, it follows that the $(3,3,4)$ is also irreducible and hence all six invariants for $N=10$ are irreducible.

\section{Invariant of Degree 12}

The $(4,4,4)$ invariant $\Omega$ is the only one of total degree 12 given in the complete system. The coefficient of $\left\{6^{2}\right\}$ in the product $[\{2\} \otimes\{6\}]^{3}$ is 30 , obtained from Young Tableaux corresponding to the following $S$-function products:

$$
\begin{aligned}
& \{62\}^{3} ;\{62\}^{2}\left\{4^{2}\right\} ;\{62\}\left\{4^{2}\right\}\{62\} ;\{62\}\left\{4^{2}\right\}\left\{42^{2}\right\} ;\{62\}\left\{42^{2}\right\}\left\{4^{2}\right\} ;\{62\}\left\{42^{2}\right\}^{2} ; \\
& \left\{4^{2}\right\}\{62\}^{2} ;\left\{4^{2}\right\}\{62\}\left\{42^{2}\right\} ;\left\{4^{2}\right\}^{3} ;\left\{4^{2}\right\}^{2}\left\{42^{2}\right\} ;\left\{4^{2}\right\}^{2}\left\{2^{4}\right\} ;\left\{4^{2}\right\}\left\{42^{2}\right\}\{62\} ; \\
& \left\{4^{2}\right\}\left\{42^{2}\right\}\left\{4^{2}\right\} ;\left\{4^{2}\right\}\left\{42^{2}\right\}^{2} ;\left\{4^{2}\right\}\left\{2^{4}\right\}\left\{4^{2}\right\} ;\left\{42^{2}\right\}\{62\}\left\{4^{2}\right\} ;\left\{42^{2}\right\}\{62\}\left\{42^{2}\right\} ; \\
& \left\{42^{2}\right\}\left\{4^{2}\right\}\{62\} ;\left\{42^{2}\right\}\left\{4^{2}\right\}^{2} ;\left\{42^{2}\right\}\left\{4^{2}\right\}\left\{42^{2}\right\} ;\left\{42^{2}\right\}^{2}\{62\} ;\left\{42^{2}\right\}^{2}\left\{4^{2}\right\} ; 3\left\{42^{2}\right\} ; \\
& \left\{42^{2}\right\}^{2}\left\{2^{4}\right\} ;\left\{42^{2}\right\}\left\{2^{4}\right\}\left\{42^{2}\right\} ;\left\{2^{4}\right\}\left\{4^{2}\right\}^{2} ;\left\{2^{4}\right\}\left\{42^{2}\right\}^{2} ;\left\{2^{4}\right\}^{3} .
\end{aligned}
$$

Reducible $(4,4,4)$ types are as follows:

$\begin{array}{lll}(4,0,0)(0,4,0)(0,0,4) \\ (4,0,0)(0,3,1)(0,1,3) & (0,4,0)(3,0,1)(1,0,3) \quad(0,0,4)(3,1,0)(1,3,0)\end{array} \quad 7$

$$
(4,0,0)(0,2,2)(0,2,2) \quad(0,4,0)(2,0,2)(2,0,2) \quad(0,0,4)(2,2,0)(2,2,0)
$$$$
(3,1,0)(1,2,1)(0,1,3) \quad(1,3,0)(2,1,1)(1,0,3) \quad(3,0,1)(1,2,1)(0,2,2)
$$$$
(3,1,0)(1,1,2)(0,2,2) \quad(1,3,0)(1,1,2)(2,0,2) \quad(3,0,1)(1,1,2)(0,3,1)
$$$$
(3,1,0)(1,0,3)(0,3,1) \quad(1,3,0)(3,0,1)(0,1,3) \quad(1,0,3)(1,2,1)(2,2,0)
$$$$
(0,3,1)(2,1,1)(2,0,2) \quad(0,1,3)(2,1,1)(2,2,0)
$$$$
(2,2,0)(1,1,2)(1,1,2) \quad(2,0,2)(1,2,1)(1,2,1)
$$$$
(2,2,0)(0,2,2)(2,0,2) \quad(0,2,2)(2,1,1)(2,1,1)
$$

7

$$
\begin{aligned}
& (2,1,1)(1,2,1)(1,1,2) \\
& (2,2,2)(2,2,2)
\end{aligned}
$$$$
\begin{array}{lll}
(3,3,2)(1,1,2) & (3,2,3)(1,2,1) & (2,3,3)(2,1,1) \\
(4,2,2)(0,2,2) & (2,4,2)(2,0,2) & (2,2,4)(2,2,0)
\end{array}
$$

Thus $v^{1}=30$ and so $v=v^{1}$, which implies that either there is no irreducible invariant of degree 12 or there exists a syzygy among the thirty reducible invariant products listed above.

If a syzygy exists it must be an expression symmetric in the coefficients of the three quadrics of the form:

$$
k_{1} \Sigma(B C)^{2}(B C a \alpha)^{2}+k_{2} \Sigma(A b c)^{2}(A \beta \gamma)^{2}+k_{3} \Phi_{123}^{2}+\Sigma k_{i}^{\prime} \Pi_{3}^{(i)}=0 \ldots(S)
$$

where $\Pi_{3}^{(i)}$ is a symmetric sum of products of three degree four invariants. $S$ can equally well be written as a linear relation between $(4,4,4)$ coefficients of generalised combinants and $(4,4,4)$ real combinants such as

$$
P(\alpha \beta \gamma)^{3}(\beta \gamma \xi) \alpha_{\lambda}
$$


and

$$
P(\alpha \beta \gamma)^{4} \text {. }
$$

Application of $\Omega_{21}$ polarisation (A3) to a $(4,4,4)$ coefficient. of (i) gives a $(5,3,4)$ invariant, while similar polarisation of (ii) yields a zero result. Hence, a syzygy among $(5,3,4)$ invariants is obtained unless $S$ is expressible solely in terms of real combinants. $S$-function analysis applied to $(5,4,3)$ reducible types gives $v=24$, viz. the coefficient of $\left\{6^{4}\right\}$ in the product

$$
[\{2\} \otimes\{5\}][\{2\} \otimes\{4\}][\{2\} \otimes\{3\}],
$$

and moreover, it is found that $v^{1}=24$ also, when the number of $(5,4,3)$ reducible invariant products is counted. Therefore, there is no syzygy among the $(5,4,3)$ invariant forms, and similarly none among the $(5,3,4)$ and accordingly, $S$ if it exists, must be expressible in terms of real combinants only, that is in terms of invariants of the combinantal forms for $N=4,6,8$ regarded as ternary in $\lambda$ (A4).

It is easy to verify that there are only two such invariants of the three ternary forms, viz. $C_{\Delta} \equiv\left(d d^{\prime} d^{\prime \prime}\right)^{4}$ and $C_{\Phi} \equiv 441 \Phi_{123}^{2}$, where

$$
d_{\lambda}^{4} \equiv d_{\lambda}^{\prime 4} \equiv d_{\lambda}^{\prime \prime 4} \equiv P p_{\lambda}^{4} \equiv\left(r r^{\prime} r^{\prime \prime} r^{\prime \prime \prime}\right)^{2} \rho_{\lambda} \rho_{\lambda}^{\prime} \rho_{\lambda}^{\prime \prime} \rho_{\lambda}^{\prime \prime \prime}
$$

and $21 \Phi_{123}=P(\alpha \beta \gamma)^{2}=\left(r r^{\prime} s s^{\prime}\right)\left(s s^{\prime} t t^{\prime}\right)\left(t t^{\prime} r r^{\prime}\right)(\rho \sigma \tau)\left(\rho^{\prime} \sigma^{\prime} \tau^{\prime}\right)$. Thus, a $(4,4,4)$ syzygy would have to assume the form: $\Phi_{123}^{2}=k C_{\Delta}$. However, $\Phi_{123}=0$ is the well-known condition that the quadric net should be pentahedral (B, p. 242), the sextic locus of its cone vertices corresponding to a Lüroth plane quartic $d_{\lambda}^{4}=0$. The cubic invariant of $d_{\lambda}^{4}$ on the other hand is $C_{\Delta}$ and its vanishing is the condition for the quartic locus $d_{\lambda}^{4}$ to be apolar to the equianharmonic envelope $\xi_{\delta}^{4}$, where $\delta=d^{\prime} d^{\prime \prime}$. The existence of syzygy $S$ is therefore impossible and so the invariant $\Omega$ is reducible.

\section{Reduction of $\Omega$}

By symmetry $\Omega$ takes the form:

$$
\begin{aligned}
\Omega & =k_{1} \sum_{3}(B C)^{2}(B C a \alpha)^{2}+k_{2} \sum_{3}(A b c)^{2}(A \beta \gamma)^{2}+\lambda \Phi_{123}^{2} \\
& +k_{3}(A b c)^{2}(B a c)^{2}(C a b)^{2}+k_{4} \sum_{3}(A b c)^{2}(A b c)^{2}(B C)^{2} \\
& +k_{5} \sum_{3}(A b c)^{2}\left[(A B)^{2} b_{\gamma}^{2}+(C A)^{2} c_{\beta}^{2}\right]+k_{6} \sum_{3}(A b c)^{2} a_{\beta}^{2} a_{\gamma}^{2}+k_{7}(B C)^{2}(C A)^{2}(A B)^{2} \\
& +k_{8} \sum_{3}(B C)^{2}(B C)^{2} a_{\alpha}^{2}+k_{9} a_{\alpha}^{2} b_{\beta}^{2} c_{\gamma}^{2}+k_{10} \sum_{3} a_{\alpha}^{2} b_{\gamma}^{2} c_{\beta}^{2}+k_{11} \sum_{2} a_{\beta}^{2} b_{\gamma}^{2} c_{\alpha}^{2} \ldots \ldots \ldots \ldots \ldots
\end{aligned}
$$

$\Omega_{21}$ polarisation of both sides of (i) will give, by equating coefficients, 24 equations in $k_{i}(i=1-11)$, since $\Omega_{21} \Phi_{123}=0$. Steps in the detailed working are summarised as follows:

$$
\begin{aligned}
2 \Omega_{21} \Omega & =a_{\beta} c_{\beta} c_{\alpha} a_{\alpha}^{\prime} a_{y}^{\prime} a_{y}+3\left(a a^{\prime} B\right)\left(c a^{\prime} B\right) c_{\alpha} b_{\alpha} b_{y} a_{y} \\
& =\frac{1}{16} a_{a}^{2} a_{\beta}^{2} c_{y}^{2}+\frac{3}{2}(A B c \gamma)(A B) c_{\alpha} b_{\alpha} b_{y} .
\end{aligned}
$$


To reduce the second term of (ii) an identity is obtained for $(A B c \gamma) b_{\alpha}$ by means of determinantal permutations, viz.

$$
\begin{array}{r}
(A b c)(B \alpha \gamma)+(A B) b_{\gamma} c_{\alpha}=-(A B c \gamma) b_{\alpha}+(A \beta \gamma) c_{\alpha}+(A B) b_{\alpha} c_{\gamma}-\dot{a}_{\beta} \dot{a}_{\alpha}^{\prime} c_{\gamma} \\
+(\dot{a} B c) \dot{a}_{\alpha}^{\prime} b_{\gamma}-c_{\beta}\left(a a^{\prime} / \alpha \gamma\right) . \ldots \ldots \ldots
\end{array}
$$

Substitution of this result in (ii) gives

$$
\begin{aligned}
& \frac{3}{2}(A B c \gamma) b_{\alpha}(A B) c_{\alpha} b_{\gamma}=\frac{1}{2}(A \beta \gamma)^{2} c_{\alpha}^{2}+\frac{3}{8}(A B)^{2} b_{\alpha}^{2} c_{\gamma}^{2}-\frac{3}{2}(A B)^{2} b_{\gamma}^{2} c_{\alpha}^{2} \\
& +\frac{3}{4}(B a c)^{2} a_{\alpha}^{2} b_{\gamma}^{2}-\frac{1}{8} a_{\alpha}^{2} a_{\beta}^{2} c_{\gamma}^{2}-\frac{1}{4} a_{\alpha}^{2} a_{\gamma}^{2} c_{\beta}^{2}-\frac{3}{2}(A b c)(B \alpha \gamma)(A B) c_{\alpha} b_{\gamma} .
\end{aligned}
$$

To obtain reduction of the last term of (iv) identity (iii) is now squared and simplification yields the result:

$$
\begin{aligned}
& (A b c)^{2}(B \alpha \gamma)^{2}+(A B)^{2} b_{\gamma}^{2} c_{\alpha}^{2}+2(A b c)(B \alpha \gamma)(A B) b_{\gamma} c_{\alpha} \\
& =(A B c \gamma)^{2} b_{\alpha}^{2}+\frac{1}{3}(A B \gamma)^{2} c_{\alpha}^{2}+\frac{1}{2}(B a c)^{2} a_{\alpha}^{2} b_{\gamma}^{2}-\frac{1}{12} a_{\alpha}^{2} a_{\beta}^{2} c_{\gamma}^{2}-\frac{1}{b} a_{\alpha}^{2} a_{\gamma}^{2} c_{\beta}^{2} .
\end{aligned}
$$

From (v), (iv) and (ii) the polarised form of $\Omega$ becomes

$$
\begin{aligned}
2 \Omega_{21} \Omega= & -\frac{3}{4}(A B c \gamma)^{2} b_{\alpha}^{2}+\frac{3}{4}(A b c)^{2}(B \alpha \gamma)^{2}+1(A \beta \gamma)^{2} c_{\alpha}^{2} \\
& +\frac{3}{8}(B a c)^{2} a_{\alpha}^{2} b_{\gamma}^{2}+\frac{3}{8}(A B)^{2} b_{\alpha}^{2} c_{\gamma}^{2}-\frac{3}{4}(A B)^{2} b_{\gamma}^{2} c_{\alpha}^{2}-\frac{1}{8} a_{\alpha}^{2} a_{\gamma}^{2} c_{\beta}^{2} .
\end{aligned}
$$

$\Omega_{21}$ polarisation of the R.H.S. of (i) now yields the following typical results, only a few of the $2 \Omega_{21} \Pi_{3}^{(i)}$ expressions being given as illustrations, since the others are easily obtained.

Terms with coefficient $k_{1}$ :

$$
\begin{gathered}
2 \Omega_{21}\left[(B C)^{2}(B C a \alpha)^{2}\right]=2(C a b)^{2}(B C a \alpha)^{2}+2(B C)^{2}(C A)^{2} b_{\alpha}^{2}+(C a b)^{2}(B C)^{2} a_{\alpha}^{2} \\
2 \Omega_{21}\left[(C A)^{2}(C A b \beta)^{2}\right]=\frac{2}{3}(C A)^{2}(C \alpha \beta)^{2}+3(C a b)^{2}(A B)^{2}(C A)^{2} \\
+(C A)^{2}(C A)^{2} a_{\beta}^{2}+(B C)^{2}(C A)^{2} b_{\alpha}^{2} \\
2 \Omega_{21}\left[(A B)^{2}(A B c \gamma)^{2}\right]=2(A B c \gamma)^{2} b_{\alpha}^{2}+2(A b c)^{2}(A B)^{2} a_{\gamma}^{2} \\
+\frac{2}{3}(A B)^{2} b_{\gamma}^{2} c_{\alpha}^{2}+\frac{1}{3}(A B)^{2} b_{\alpha}^{2} c_{\gamma}^{2}
\end{gathered}
$$

Coefficient $k_{2}$ :

$$
\begin{aligned}
2 \Omega_{21}\left[(A b c)^{2}(A \beta \gamma)^{2}\right] & =(A \beta \gamma)^{2} c_{\alpha}^{2}+(A b c)^{2}(B \alpha \gamma)^{2}+3(A b c)^{2}(A B)^{2} a_{\gamma}^{2} \\
2 \Omega_{21}\left[(B a c)^{2}(B \alpha \gamma)^{2}\right] & =2(A b c)^{2}(B \alpha \gamma)^{2}+(B a c)^{2} a_{\alpha}^{2} b_{\gamma}^{2}+2(B a c)^{2} b_{\alpha}^{2} a_{\gamma}^{2} \\
2 \Omega_{21}\left[(C a b)^{2}(C \alpha \beta)^{2}\right] & =(C A)^{2}(C \alpha \beta)^{2}+3(C a b)^{2}(B C a \alpha)^{2} .
\end{aligned}
$$

Coefficient $k_{3}$ :

$$
\begin{array}{lcc}
2 \Omega_{21}\left[(A b c)^{2}(B a c)^{2}(C a b)^{2}\right]= & (B a c)^{2}(C a b)^{2} c_{\alpha}^{2}+2(A b c)^{2}(A b c)^{2}(C a b)^{2} \\
\text { Coefficient } k_{11}: & \vdots & +(A b c)^{2}(B a c)^{2}(A C)^{2}
\end{array}
$$

$$
2 \Omega_{21}\left[a_{\beta}^{2} b_{\gamma}^{2} c_{\alpha}^{2}+a_{\gamma}^{2} \dot{b}_{\alpha}^{2} c_{\beta}^{2}\right]=3(A B)^{2} b_{\gamma}^{2} c_{\alpha}^{2}+a_{\beta}^{2} a_{\gamma}^{2} c_{\alpha}^{2}+a_{\alpha}^{2} a_{\gamma}^{2} c_{\beta}^{2}+3(B a c)^{2} a_{\gamma}^{2} b_{\alpha}^{2}
$$


Comparison of coefficients of like terms in (vi) and the above polar forms give 24 equations in the 11 unknown $k_{i}$, which are found to be consistent and yield solutions

$$
k_{1}=-\frac{3}{8}, k_{2}=\frac{1}{4}, k_{3}=\mu, k_{4}=-\mu, k_{5}=\mu, k_{6}=-\mu, k_{7}=\frac{1}{2}\left(\frac{9}{8}-\mu\right),
$$

$k_{8}=\frac{1}{4}\left(\frac{3}{8}-\mu\right), k_{9}=-\frac{1}{12}\left(\frac{1}{8}+\mu\right), k_{10}=\frac{1}{3}\left(\frac{1}{8}+\mu\right), k_{11}=-\frac{1}{3}\left(\frac{1}{2}+\mu\right)$, where $\mu$ is a parameter taken equal to $k_{3}$.

Substitution of these values in (i) produces the identity

$$
E_{\Omega} \equiv \lambda \Phi_{123}^{2}+\mu E_{\Delta}
$$

where

$$
\begin{aligned}
& E_{\Omega}=\Omega+\frac{3}{8} \sum_{3}(B C)^{2}(B C a \alpha)^{2}-\frac{1}{4} \sum_{3}(A b c)^{2}(A \beta \gamma)^{2}-\frac{9}{16}(B C)^{2}(C A)^{2}(A B)^{2} \\
&-\frac{3}{32} \sum_{3}(B C)^{2}(B C)^{2} a_{\alpha}^{2}+\frac{1}{96} a_{\alpha}^{2} b_{\beta}^{2} c_{\gamma}^{2}-\frac{1}{24} \sum_{3} a_{\alpha}^{2} b_{\gamma}^{2} c_{\beta}^{2}+\frac{1}{6} \sum_{2} a_{\beta}^{2} b_{\gamma}^{2} c_{\alpha}^{2}
\end{aligned}
$$

and

$$
\begin{array}{r}
E_{\Delta}=(A b c)^{2}(B a c)^{2}(C a b)^{2}-\sum_{3}(A b c)^{2}(A b c)^{2}(B C)^{2}+\sum_{3}(A b c)^{2}\left[(A B)^{2} b_{\gamma}^{2}+(C A)^{2} c_{\beta}^{2}\right] \\
-\sum_{3}(A b c)^{2} a_{\beta}^{2} a_{\gamma}^{2}-\frac{1}{2}(B C)^{2}(C A)^{2}(A B)^{2}-\frac{1}{4} \sum_{3}(B C)^{2}(B C)^{2} a_{\alpha}^{2}-\frac{1}{12} a_{\alpha}^{2} b_{\beta}^{2} c_{\gamma}^{2} \\
+\frac{1}{3} \sum_{3} a_{\alpha}^{2} b_{\gamma}^{2} c_{\beta}^{2}-\frac{1}{3} \sum_{2} a_{\beta}^{2} b_{\gamma}^{2} c_{\alpha}^{2}
\end{array}
$$

Also since $\Omega$ is reducible there is an identity connecting real combinants of the form

$$
C_{\Omega} \equiv l C_{\Phi}+m C_{\Delta}
$$

where $C_{\Phi}, C_{\Delta}$ have been defined in $\S 5$ and

$$
\begin{aligned}
C_{\Omega} & =\left(r s^{\prime} s^{\prime \prime} s^{\prime \prime}\right)\left(t s^{\prime} s^{\prime \prime} s^{m}\right)\left(t r^{\prime} r^{\prime \prime} r^{m}\right)\left(s r^{\prime} r^{\prime \prime} r^{\prime \prime}\right)\left(s t^{\prime} t^{\prime \prime} t^{\prime \prime}\right)\left(r t^{\prime} t^{\prime \prime} t^{\prime \prime}\right)(\rho \sigma \tau)\left(\rho^{\prime} \sigma^{\prime} \tau^{\prime}\right)\left(\rho^{\prime \prime} \sigma^{\prime \prime} \tau^{\prime \prime}\right) \\
& =6 P^{(3)}\left(a b^{\prime} b^{\prime \prime} b^{\prime \prime}\right)\left(c b^{\prime} b^{\prime \prime} b^{\prime \prime}\right)\left(c a^{\prime} a^{\prime \prime} a^{\prime \prime}\right)\left(b a^{\prime} a^{\prime \prime} a^{\prime \prime}\right)\left(b c^{\prime} c^{\prime \prime} c^{\prime \prime}\right)\left(a c^{\prime} c^{\prime \prime} c^{\prime \prime}\right),
\end{aligned}
$$

$P^{(3)}$ denoting the sum of terms obtained by independent permutation of the elements in each of the three groups: $a^{\prime}, b^{\prime}, c^{\prime} ; a^{\prime \prime}, b^{\prime \prime}, c^{\prime \prime} ; a^{m}, b^{m}, c^{m}$ with the usual sign convention. It is found that the term $16 \Omega$ occurs in the expansion of $C_{\Omega}$ and so $E_{\Omega}=\frac{1}{16} C_{\Omega}$.

Similarly, $C_{\Delta}=6 P^{(3)}\left(a a^{\prime} a^{\prime \prime} a^{\prime \prime}\right)^{2}\left(b b^{\prime} b^{\prime \prime} b^{\prime \prime}\right)^{2}\left(c c^{\prime} c^{\prime \prime} c^{\prime \prime}\right)^{2}$ which shows the coefficient of $a_{\alpha}^{2} b_{\beta}^{2} c_{\gamma}^{2}$ to be 6 and so $E_{\Delta}=-\frac{1}{72} C_{\Delta}$, while $C_{\Phi}=441 \Phi_{123}^{2}$ in similar fashion.

Identity (vii) may therefore be written as

$$
\frac{1}{16} C_{\Omega}=\frac{\lambda}{441} C_{\Omega}-\frac{\mu}{72} C_{\Delta}
$$

Expression of (ix) in terms of $r, s, t, \rho, \sigma, \tau$ and equivalent symbols enables the numerical values of $\lambda, \mu$ to be determined by comparison of coefficients 
of like terms. It is found that $\lambda=0, \mu=-\frac{9}{2}$ and finally by substitution of those values in (vii) the reduction formula for $\Omega$ is:

$$
\begin{aligned}
\Omega= & -\frac{1}{8}\left[3 \sum_{3}(B C)^{2}(B C a \alpha)^{2}-2 \sum_{3}(A b c)^{2}(A \beta \gamma)^{2}\right] \\
- & -\frac{9}{2}\left[(A b c)^{2}(B a c)^{2}(C a b)^{2}-\sum_{3}(A b c)^{2}(A b c)^{2}(B C)^{2}\right. \\
& \left.+\sum_{3}(A b c)^{2}\left\{(A B)^{2} b_{\gamma}^{2}+(C A)^{2} c_{\beta}^{2}\right\}-\sum_{3}(A b c)^{2} a_{\beta}^{2} a_{\gamma}^{2}\right] \\
& +\frac{3}{32}\left[30(B C)^{2}(C A)^{2}(A B)^{2}+13 \sum_{3}(B C)^{2}(B C)^{2} a_{\alpha}^{2}\right] \\
& +\frac{1}{96}\left[35 a_{\alpha}^{2} b_{\beta}^{2} c_{\gamma}^{2}-140 \sum_{3} a_{\alpha}^{2} b_{\gamma}^{2} c_{\beta}^{2}+128 \sum_{2} a_{\beta}^{2} b_{\gamma}^{2} c_{\alpha}^{2}\right] .
\end{aligned}
$$

\section{Invariants of Degree 14}

The $(6,6,2)$ invariant type is found to be irreducible, there being 5 relevant Young Tableaux corresponding to the number $v$ of $\left\{7^{4}\right\} S$-functions in the product $[\{2\} \otimes\{6\}]^{2}[\{2\} \otimes\{2\}]$, viz.

$$
\left\{6^{2}\right\}^{2}\left\{2^{2}\right\} ; \quad\{642\}^{2}\{4\} ; \quad 2\{642\}^{2}\left\{2^{2}\right\} ; \quad\left\{4^{2} 2^{2}\right\}^{2}\left\{2^{2}\right\},
$$

whereas $v^{\prime}=4$ arising from the reducible types

$$
\begin{gathered}
(2,2,0)(4,4,2) ; \quad(2,2,2)(4,0,0)(4,0,0) ;(2,2,2)(3,1,0)(1,3,0) ; \\
(2,2,2)(2,2,0)(2,2,0) .
\end{gathered}
$$

Since $(6,6,2)$ is irreducible it follows that $P(\alpha \beta \gamma)^{2}(q r \xi)^{4}$, the combinantal form in Table (i) is applicable and so all the invariants of degree 14 are irreducible.

\section{Invariants of Degree 18}

There are three invariants of degree 18 , all of partial degrees $(6,6,6)$ and accordingly they must be real combinants. The calculation of $v$ and $v^{\prime}$ at this stage involves a high degree of complexity and so the relevant results are merely summarised. From a consideration of the term $\left\{9^{4}\right\}$ in $[\{2\} \otimes\{6\}]^{3}$ it is found that $v=75$, while a count of reducible types gives $v^{\prime}=81$. Thus, further analysis is required to determine whether or not syzygies exist among the latter.

The total number of tableaux corresponding to invariants of the various partial degrees are

$\begin{array}{rrrrrr}(14,2,2) & 1 & (10,6,2) & 7 & (8,8,2) & 9 \\ (13,3,2) & 1 & (10,5,3) & 12 & (8,7,3) & 21 \\ (12,4,2) & 3 & (10,4,4) & 19 & (8,6,4) & 43 \\ (12,3,3) & 3 & (9,7,2) & 7 & (8,5,5) & 47 \\ (11,5,2) & 4 & (9,6,3) & 18 & (7,7,4) & 44 \\ (11,4,3) & 7 & (9,5,4) & 29 & (7,6,5) & 60 \\ & & & & (6,6,6) & 75\end{array}$

E.M.S. - $\mathbf{P}$ 
belonging to combinantal forms of types

$$
\begin{aligned}
& \left\{14,2^{2}\right\}+2\{12,4,2\}+\{11,5,2\}+\{11,4,3\}+3\{10,6,2\}+\{10,5,3\}+4\left\{10,4^{2}\right\} \\
& +3\{9,6,3\}+2\{9,5,4\}+2\{8,8,2\}+\{8,7,3\}+5\{8,6,4\}+\{7,6,5\}+3\left\{6^{3}\right\} .
\end{aligned}
$$

Moreover, the combinantal forms built up from reducible concomitants account for all of these and in addition, types $\{8,6,4\},\{7,6,5\}$ and $\left\{6^{3}\right\}$. This agrees with the result $v^{\prime}=81$ for $\left\{6^{3}\right\}$, as $\{8,6,4\}$ contains 3 such forms and $\{7,6,5\}, 2$. Hence, the $(6,6,6)$ invariants are all reducible combinants and are apparently connected by a syzygy.

\section{Acknowledgment}

I should like to acknowledge my indebtedness to Dr J. A. Todd for his generosity in providing me with the detailed results given in $\S \mathbf{8}$ above for the numbers of tableaux and combinantal forms associated with the various degree 18 types, and also for his interest and encouragement freely given in the work as a whole.

\section{REFERENCES}

(A) T. Scort, Combinantal forms of a quadric net applied to irreducible concomitant systems, Proc. Edin. Math. Soc., 12 (1961), 189-195.

(B) G. SALmon, Analytic Geometry of Three Dimensions (revised by A. P. Rogers, Dublin, 1928).

Department of Mathematics

Heriot-WatT COLLEgE

EDINBURGH 